\title{
Changes of dayside auroral distribution caused by a solar wind pressure pulse and associated interplanetary magnetic field disturbances
}

\author{
A. Kozlovsky ${ }^{1}$, M. Meurant ${ }^{2,3}$, and T. Turunen ${ }^{1}$ \\ ${ }^{1}$ Sodankylä Geophysical Observatory, Sodankylä, Finland \\ ${ }^{2}$ Institute for Space Research, University of Calgary, Calgary, Canada \\ ${ }^{3}$ Institut d'Astrophysique et de Géophysique, Université de Liège, Liège, Belgium
}

Received: 3 January 2007 - Revised: 16 March 2007 - Accepted: 30 March 2007 - Published: 8 May 2007

\begin{abstract}
Global auroral images from the IMAGE satellite were used to study statistically changes of the dayside aurora spatial distribution after an abrupt solar wind pressure increase, or so-called "Sudden Impulse" (SI). Contributions from IMF changes associated with a SI were also investigated. The effects of the IMF and pressure variations were separated using a multi-factor correlation analysis. The first prominent effect due to pressure increase is the auroral intensification equatorward of the middle dayside oval within $6 \mathrm{~min}$ after a SI occurred. This is consistent with the midday sub-auroral patches. The second effect due to pressure increase is the auroral intensification at high latitudes in the vicinity of the polar cap boundary. For the first $6 \mathrm{~min}$ the auroral intensification is most prominent in the postnoon sector. Later on (6-20 $\mathrm{min})$ the intensification occurs in the prenoon sector. The most obvious effect of IMF changes is the "IMF $B y$ " effect, an intensification (fading) of the most poleward auroral forms when IMF $B_{y}$ becomes negative (positive). This effect occurs 6-20 min after changes in the interplanetary medium. Such an effect is consistent with the IMF $B_{y}$-related system of field-aligned currents. No significant motion of the dayside auroral oval was observed associated with IMF $B_{z}$ variations. This can be explained by a response time to IMF $B_{z}$ changes larger than $20 \mathrm{~min}$.
\end{abstract}

Keywords. Ionosphere (Particle precipitation) - Magnetospheric physics (Auroral phenomena; Solar windmagnetosphere interactions)

\section{Introduction}

In the last decade, extensive studies of the auroral oval large-scale dynamics have become possible due to the global ultraviolet (UV) imagers on the Polar and Imager

Correspondence to: A. Kozlovsky

(alexander.kozlovsky@oulu.fi) for Magnetopause-to-Aurora Global Exploration (IMAGE) satellites launched respectively in 1996 and 2000. The auroral oval and polar cap ionosphere can be considered as a screen on which the magnetosphere is mapped. Owning to that, auroral imagers are very useful to study global magnetospheric response to interplanetary changes. In particular, UV satellite images were used to investigate the auroral oval response to Solar Wind Pressure (SWP) changes (e.g., Zhou and Tsurutani, 1999; Brittnacher et al., 2000; Boudouridis et al., 2003; Meurant et al., 2003, 2004; Kozlovsky et al., 2005; Lyons et al., 2005).

It has been found that solar wind pressure increase (referred below as a Sudden Impulse (SI) or "shock") leads to intensification of the whole auroral oval. The intensification first occurs in the dayside and then propagates to the nightside. An important problem is the propagation speed and wave modes of the magnetospheric disturbances caused by the SI. Two types of the disturbance propagation were observed. The first type, "fast response", was observed as a nearly simultaneous (within 2 min after the SI) intensification of the whole auroral oval (e.g., Boudouridis et al., 2003; Meurant et al., 2003). In this case, the precipitation increase was probably launched by fast Alfvén (magnetoacoustic) waves propagating across magnetic flux tubes. The second type, "slower response", which takes 10-20 min, was observed as an auroral brightening propagating from noon to both west and east with a speed of 6 to $11 \mathrm{~km} / \mathrm{s}$ (Zhou and Tsurutani, 1999). Such a propagation speed corresponds to typical flow speed in the magnetosheath and can be explained by tail-ward propagation of the solar wind disturbance along the magnetopause. The most frequently discussed issues were the comparison of intense nightside shock-induced precipitation with substorm activity and the mechanisms for substorm triggering by a SI (e.g., Zhou and Tsurutani, 2001; Meurant et al., 2005; Lyons et al., 2005). However, less attention was paid to the dayside auroral response to a SI. In particular, the motion of dayside auroral

Published by Copernicus GmbH on behalf of the European Geosciences Union. 
oval and its boundaries can be an important indicator of the magnetospheric disturbances caused by the SI (see, e.g., Kozlovsky et al., 2005).

SI-related motion of dayside auroral oval and its boundaries was studied in several papers. Brittnacher et al. (2000) reported one case of Polar UVI observation when a pressure pulse caused the equatorward shift of the low-latitude boundary of the dayside aurora. Later on, Liou et al. (2002) presented 3 cases of a sudden brightening of the auroral patch in the midday (10:00-14:00 MLT) sub-auroral zone (above $65^{\circ}$ MLAT) caused by the SI. These patches were named Midday Sub-auroral Patches (MSPs). After brightening, the MSP widened and/or moved equatorward.

Boudouridis et al. (2003) have examined three SI events, one case with steady southward IMF, one with an IMF $B_{z}$ simultaneous northward turning, and one under near-zero IMF $B_{z}$. Data from the Defense Meteorological Satellite Program (DMSP) low-altitude satellites were also used in their study. All cases showed a significant change in the auroral oval location, its size and its intensity in response to solar wind pressure variations. For the case occurring under steady southward IMF conditions, the most prominent observed features were an increase of the auroral zone luminosity band and a decrease of the polar cap size after a solar wind dynamic pressure increase. For the two other cases, with IMF $B_{z}$ simultaneous northward turning and IMF $B_{z}$ near zero, a smaller response was observed.

One case of simultaneous IMAGE satellite and groundbased optical observations during the SI was studied by Kozlovsky et al. (2005). They have shown that after the SI, new discrete auroral forms appeared in the poleward part of the auroral oval so that the dayside oval moved poleward. This motion of the auroral oval was caused by the redistribution of luminosity within the oval and was not associated with motion of the poleward boundary of diffuse luminosity.

In total, eight events have been described in the abovementioned papers and different kinds of auroral responses to the SI were observed. Since the number of studied cases is not large enough, it is not clear how common the reported features are. In particular, data on the motion of poleward auroral oval boundary still look controversial. However, together with the SI, other factors may influence the auroras, which make each event unique to some extent. Obviously, preconditioning of the magnetosphere can play an important role in the response (e.g., Boudouridis et al., 2003). On the other hand, solar wind pressure changes are, as a rule, associated with simultaneous IMF changes, which can also affect the dayside aurora, especially in the most poleward part of the oval. For instance, the auroral oval motion following IMF $B_{z}$ changes was observed by Polar UVI in both prenoon (Kozlovsky et al., 2003a) and post-noon (Kozlovsky and Kangas, 2001) MLT sectors. The IMF $B_{y}$ control of dayside high-latitude auroras was demonstrated by Trondsen et al. (1999). However, possible contribution of the associated
IMF changes was usually ignored in the studies of shockinduced auroral effects.

The key question of the present paper is: what is the "pure" SWP contribution to the shock-induced changes of the dayside auroral oval position and shape? In other words, we attempt to separate effects of IMF and SWP changes. We only consider the dayside oval because the IMF effects are expected to be most significant there.

\section{Observations and data presentation}

For this study, we consider the time period from 1 May 2000 to 31 December 2002, during which the northern polar region was optimally viewed by the far ultraviolet (FUV) imaging system on the IMAGE satellite (Mende et al., 2000a). We use the Wideband Imaging Camera (WIC) (Mende et al., 2000b) data obtained every $2 \mathrm{~min}$ with $10 \mathrm{~s}$ image exposure. There are 20 events selected for which the entire auroral oval was observed during solar wind pressure increases. These events are listed in Table 1.

The Advanced Composition Explorer (ACE) spacecraft located upstream in the solar wind (at about $250 R_{E}$ in front of the magnetosphere) monitored the solar wind speed and plasma density and the IMF conditions. To characterize the events, we use the IMF components and SWP medianaveraged over $20 \mathrm{~min}$ before and after the pressure jumps. This average time interval corresponds to a typical response time of the dayside magnetosphere to interplanetary changes (e.g., Khan and Cowley, 1999). In addition to ACE, data from the Wind satellite located in the solar wind somewhat apart from the Earth-Sun line are available. Using the arrival times of disturbances to the locations of these two satellites, we determined the SWP front tilt with respect to the EarthSun line. This gives the Magnetic Local Time (MLT) sector where the SWP front hits the magnetosphere. The interplanetary changes characteristics are presented in Table 1. In most (67\%) of the studied cases, the SWP front hit the magnetosphere near noon (11:00-13:00 MLT).

The SI arrival time in the magnetosphere $\left(t_{0}\right)$ is identified as the start of increase in the horizontal magnetic field $(\mathrm{H})$ component measured at near-equatorial observatories Guimar (GUI, 28.3 N, 16.4E), Kanoya (KNY, 31.4 N, 130.9 E), Honolulu (HON, 21.32 N, 158.1 E), and Tondano (TND, 1.3 N, 124.9E). For each SI case, we consider the WIC auroral data $10 \mathrm{~min}$ prior to $t_{0}$ until $20 \mathrm{~min}$ after $t_{0}$.

As an example, Fig. 1 presents the event of 7 December (day 342) 2000. Shown on the left are the interplanetary parameters measured by the ACE spacecraft. The SWP jump occurred at the ACE location at 06:23 UT and arrived to the magnetosphere in about $1 \mathrm{~h}$ causing a ground magnetic disturbance in Guimar at $t_{0}=07: 32$ UT. A sequence of WIC frames (in the frame of magnetic latitude and MLT) shows the auroral oval dynamics from $t_{0}-10 \mathrm{~min}$ to $t_{0}+20 \mathrm{~min}$. 
Table 1. Parameters of events.

\begin{tabular}{|c|c|c|c|c|c|c|c|c|c|c|c|c|c|c|c|c|}
\hline \multicolumn{3}{|c|}{ Event } & \multicolumn{4}{|c|}{ Pressure pulse } & \multicolumn{4}{|c|}{ IMF change } & \multicolumn{6}{|c|}{ MLAT of the noon mid oval and PAB } \\
\hline $\mathrm{N}$ & Year & day & UT & & w & MLT & IM & $B_{y}$ & IM & $B_{z}$ & $-10-0 \mathrm{~min}$ & $0-6 \mathrm{~min}$ & $6-20 \mathrm{~min}$ & $-10-0 \mathrm{~min}$ & $0-6 \min$ & $6-20 \mathrm{~min}$ \\
\hline 1 & 2000 & 192 & $06: 37$ & 1.8 & 4.6 & - & 6.9 & 15.6 & -6.5 & -7.6 & 74.4 & 73.4 & 73.0 & 82.5 & 83.9 & 84.5 \\
\hline 2 & 2000 & 208 & $18: 57$ & 2.7 & 5.8 & $10: 00$ & 2.4 & 3.5 & -3.8 & -8.7 & 71.2 & 71.9 & 71.6 & 78.6 & 81.5 & 80.2 \\
\hline 3 & 2000 & 261 & $00: 41$ & 2.9 & 13.4 & $13: 00$ & 14.0 & -3.7 & -2.9 & -0.5 & 69.7 & 68.9 & 69.4 & 83.6 & 82.0 & 83.5 \\
\hline 4 & 2000 & 277 & $00: 53$ & 1.9 & 5.0 & $13: 00$ & 2.3 & 5.6 & -1.6 & -3.6 & 68.4 & 68.2 & 68.2 & 83.1 & 82.6 & 82.5 \\
\hline 5 & 2000 & 309 & $02: 21$ & 3.1 & 14.9 & $13: 00$ & -2.9 & -12.6 & 5.6 & 8.0 & 70.3 & 71.2 & 71.9 & 76.6 & 77.7 & 81.6 \\
\hline 6 & 2000 & 311 & $09: 48$ & 0.9 & 3.4 & $10: 00$ & 1.3 & 3.5 & -4.4 & -9.6 & 69.1 & 71.0 & 71.1 & 74.4 & 80.5 & 81.1 \\
\hline 7 & 2000 & 331 & $11: 58$ & 3.1 & 11.9 & $14: 00$ & 5.2 & 7.8 & 2.1 & 8.7 & 79.4 & 76.9 & 77.7 & 80.6 & 81.4 & 82.4 \\
\hline 8 & 2000 & 342 & $07: 32$ & 2.4 & 5.9 & $15: 00$ & 6.4 & 4.4 & 2.2 & 1.8 & 71.4 & 71.0 & 73.2 & 79.1 & 79.2 & 80.0 \\
\hline 9 & 2001 & 10 & $16: 20$ & 1.1 & 3.8 & $13: 00$ & 0.0 & -1.1 & -3.3 & -5.9 & 70.3 & 71.1 & 71.1 & 79.7 & 80.5 & 80.3 \\
\hline 10 & 2001 & 19 & $03: 17$ & 0.6 & 2.6 & $12: 00$ & 5.1 & 0.6 & 3.6 & 1.9 & 73.5 & 73.2 & 73.7 & 79.7 & 78.6 & 79.6 \\
\hline 11 & 2001 & 62 & $11: 20$ & 1.4 & 3.5 & 08:00 & 2.3 & 6.4 & -3.4 & -2.5 & 71.5 & 71.8 & 71.5 & 80.7 & 80.7 & 81.1 \\
\hline 12 & 2001 & 108 & $00: 48$ & 1.4 & 10.4 & $12: 00$ & -2.6 & -5.6 & -3.4 & -16.1 & 70.1 & 69.4 & 70.0 & 79.2 & 78.4 & 78.8 \\
\hline 13 & 2001 & 118 & 05:01 & 1.3 & 9.6 & $12: 00$ & 7.4 & 19.8 & 0.0 & 5.2 & - & 74.3 & 73.6 & - & 84.5 & 85.6 \\
\hline 14 & 2001 & 294 & $16: 48$ & 2.4 & 14.3 & 09:00 & -4.0 & 13.9 & -6.0 & -13.7 & 71.9 & 72.4 & 70.4 & 77.6 & 79.0 & 75.1 \\
\hline 15 & 2001 & 298 & 08:50 & 1.2 & 7.0 & $12: 00$ & -0.3 & -1.1 & 0.5 & 4.3 & - & 75.1 & 74.7 & - & 76.6 & 77.7 \\
\hline 16 & 2001 & 323 & $18: 16$ & 1.2 & 4.3 & $11: 00$ & 4.6 & 10.7 & 0.2 & 3.0 & 70.8 & 72.1 & 72.5 & 81.0 & 82.1 & 82.1 \\
\hline 17 & 2001 & 364 & $20: 10$ & 2.0 & 8.3 & $12: 00$ & -10.8 & -21.2 & 3.1 & 9.0 & - & 74.5 & 75.9 & - & 82.0 & 81.7 \\
\hline 18 & 2002 & 8 & 01:55 & 1.2 & 4.8 & - & -5.8 & 2.8 & -3.5 & 2.1 & 72.3 & 71.7 & 71.9 & 76.4 & 76.9 & 76.2 \\
\hline 19 & 2002 & 77 & $13: 23$ & 2.8 & 16.1 & $12: 00$ & 2.4 & 6.7 & 0.8 & 5.0 & - & 76.4 & 77.7 & - & 85.6 & 85.1 \\
\hline 20 & 2002 & 113 & $04: 49$ & 1.7 & 8.8 & 11:00 & 5.6 & 6.9 & -4.0 & 3.8 & - & 73.1 & 73.0 & - & 85.2 & 84.4 \\
\hline
\end{tabular}
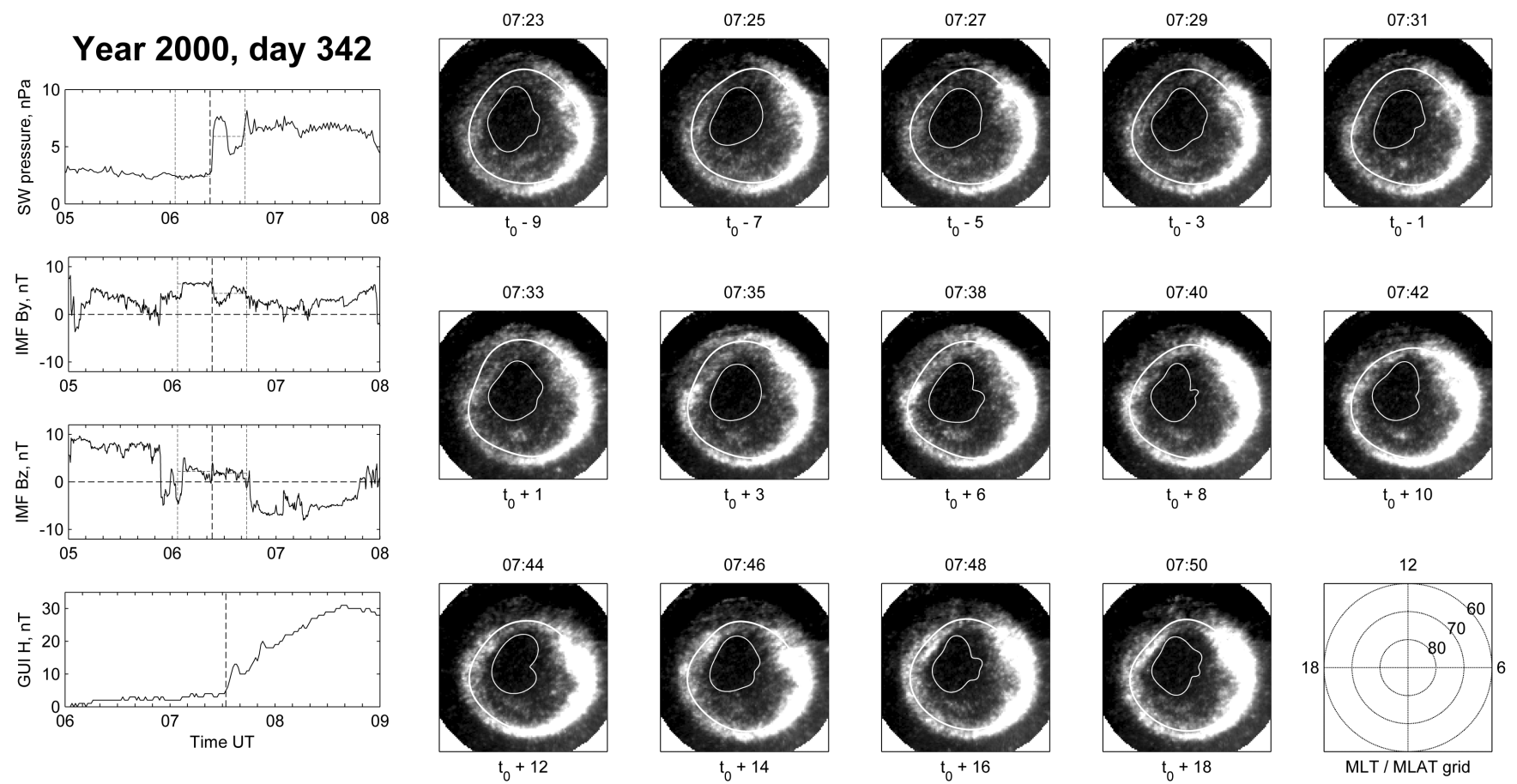

Fig. 1. A sequence of WIC frames for event \#8, where the calculated mid oval and PAB are indicated by thick and thin white lines, respectively. The WIC frames were mapped into the frame of AACGM latitude and magnetic local time (MLT). Left panel shows the interplanetary conditions and the magnetogram from near-equatorial observatory Guimar. The SI time is indicated by dashed line.

The images are presented in the Altitude Adjusted Corrected Geomagnetic (AACGM) coordinate system (for which any two points connected by a magnetic field line have the same AACGM coordinates). The altitude of UV luminosity is assumed to be of $130 \mathrm{~km}$. Day glow was subtracted from all frames. After that, for each event an averaged lumi- nosity value of the polar cap (within the circle of $85 \mathrm{deg}$.), $I_{P C}$, with the standard deviation $\left(\Delta I_{P C}\right)$ was calculated. Then, for a quantitative analysis, using a method similar to one suggested by Kozlovsky et al. (2005), two characteristics of the large-scale auroral distribution were determined. 
The first characteristic is the mid auroral oval represented by a curve given as the harmonic function of magnetic local time $M_{L T}$ :

$$
R_{A}=\sum_{k=0}^{5}\left(a_{k}^{c} \cos \left(2 \pi k \frac{M_{L T}}{24}\right)+a_{k}^{s} \sin \left(2 \pi k \frac{M_{L T}}{24}\right)\right)
$$

where $R_{A}$ is the AACGM co-latitude (i.e., the distance from the geomagnetic pole) of the mid auroral oval. Coefficients $a_{k}$ were calculated by minimizing the following integral:

$$
\begin{aligned}
& \iint\left(R_{A}\left(M_{L T}\right)-R\right)^{2} \\
& \quad \times\left\{\begin{array}{l}
\left(I\left(R, M_{L T}\right)-I_{0}\right), \text { if } I>I_{0} \\
0, \text { if } I<I_{0}
\end{array} d R d M_{L T},\right.
\end{aligned}
$$

where $R$ and $M_{L T}$ are the co-latitude and local time of a pixel in the WIC frame, and $I$ is its UV intensity. The threshold intensity $I_{0}$ (selected as $I_{P C}+\Delta I_{P C}$ ) was used to reject the background low-intensity pixels both poleward and equatorward of the auroral oval. Integration was performed over an area poleward of $60^{\circ}$ AACGM latitude. Minimizing integral (2) means that at each local time the integrated UV power values poleward and equatorward of the mid oval are equal. In each frame, the latitude of the mid auroral oval is determined with accuracy of the order of $0.5^{\circ}$, which corresponds to the spatial resolution of the WIC imager.

The second characteristic, referred below as the Poleward Auroral Boundary (PAB), is defined as a contour curve corresponding to the UV intensity of $I_{0}=I_{P C}+\Delta I_{P C}$. The experimental PAB location points were approximated by a smooth curve similar to Eq. (1):

$$
R_{P A B}=\sum_{k=0}^{5}\left(b_{k}^{c} \cos \left(2 \pi k \frac{M_{L T}}{24}\right)+b_{k}^{s} \sin \left(2 \pi k \frac{M_{L T}}{24}\right)\right) .
$$

Coefficients $b_{k}$ were calculated for each image using the least-square method. The latitude of the PAB is determined with an average accuracy of $1.3^{\circ}$.

Before describing the observations, we should make some remarks about the meaning of the introduced parameters. To characterize the poleward part of the auroral oval, we consider a contour curve corresponding to a certain brightness threshold. One should emphasize that the contour line of the UV intensity (PAB) may not coincide with the magnetospheric open-closed boundary (in particular, dayside auroras may be on open magnetic field lines associated with cusp/mantle precipitation) or with a boundary of a particle precipitation magnetospheric region. Indeed, the PAB position strongly depends on the selected threshold value. For an individual case, if there is a sharp difference between the Polar Cap (PC) and auroral oval intensities, the threshold may be carefully selected in such a way that the obtained contour curve more or less corresponds to the boundary of diffuse luminosity (e.g., Kozlovsky et al., 2005). In this case, the curve can be defined as the Poleward auroral Oval Boundary (POB). In an ideal POB case, the threshold should be as close as possible to the mean PC intensity (i.e., $I_{0} \approx I_{P C}$ ). However, in many cases the dayside oval boundary is smooth and obscure. Its position may depend on the dayglow removal procedure, and moreover, some week auroras may appear in the polar cap (although we do not consider cases with bright transpolar arcs). Consequently, determination of the POB becomes ambiguous. In the present study, we introduce the auroral boundary $(\mathrm{PAB})$ at the selected threshold $I_{0}=I_{P C}+\Delta I_{P C}$, which is obviously larger than the PC intensity. To emphasize the difference from POB, we use the abbreviation PAB. The PAB position itself may be a useless quantity, but the motion of PAB is a useful characteristic of auroral dynamics in the vicinity of the polar cap boundary. Poleward motion of the PAB indicates intensification (or/and generation of new auroral forms) in the vicinity of PC boundary, whereas equatorward motion of the PAB means fading of pre-existing most high-latitude auroras (the latter may be caused by an equatorward shift of the open-closed boundary).

Distinctly from the PAB, the position of the mid auroral oval as determined by Eqs. (1) and (2) is a quite certain and reliable quantity. Motion of the mid auroral oval occurs due to spatial re-distribution of auroral intensity. For instance, equatorward shift of the mid auroral oval may be due to precipitation increase or new precipitation in the equatorial part of the oval (or equatorward of the main auroral oval), or fading of pre-existing precipitation in the poleward part of the oval. The latter can be also seen as PAB motion toward equator.

In frames in Fig. 1, the calculated mid oval and PAB are indicated respectively by thick and thin white lines. In the following sections we focus on the motion of the PAB and mid oval during the SI.

\section{Correlation analysis}

We consider response of the dayside oval in the pre-noon (06:00-10:00 MLT), noon (10:00-14:00 MLT), and postnoon (14:00-18:00 MLT) sectors. For each sector, we calculated the latitudes of the mid oval and PAB averaged over the MLT sector and median-averaged over 10 min just prior to the SI, over 6 min just after the SI and 6-20 min after the SI. Standard deviations of the locations at 10, 6, and $14 \mathrm{~min}$, respectively (with a linear trend subtracted in the last two values implying changes caused by the SI), were also calculated to characterize the uncertainty of the averaged latitudes. These standard deviations are used below to estimate errors of the mid-oval and PAB shifts caused by the interplanetary disturbances. These averaged latitudes of the mid oval and PAB for 10:00-14:00 MLT sector are presented in the right part of Table 1 . In 5 of 20 cases, the auroral intensity prior to the SI was weak making impossible a reliable identification of the auroral oval position. 
In each of three MLT sectors, shifts in the mid oval and PAB positions (referred below as Do and Db, respectively) were calculated for the first $6 \mathrm{~min}$ (i.e., Do6=LatOval(0:6) - LatOval(-10:0)) and the next 6-20 min (i.e., Do20=LatOval(6:20)-LatOval(0:6)). We suppose that the shifts occurring during the first 6 min (i.e., Do6 and Db6) can be considered, to some extent, as a manifestation of the "fast" response, whereas the motion observed from 6 to 20 min (Do20 and Db20) after the SI should be attributed to the "slow" response. Thus, the motion of the auroral oval in each event is characterized by 12 parameters, i.e., 4 shifts $\mathrm{D}(\mathrm{o} / \mathrm{b})(6 / 20)$ in three MLT sectors (pre-noon, noon, and post-noon). On the other hand, each event is characterized by the interplanetary changes $\Delta \mathrm{Psw}, \Delta B_{x}, \Delta B_{y}$, and $\Delta B_{z}$, which we determined as differences between the values averaged over $20 \mathrm{~min}$ after and prior to the SWP jumps (given in Table 1). Our next purpose is to investigate how the 12 parameters of the oval are controlled by the interplanetary changes. Multi-factor linear regression analysis is performed to achieve this goal.

We assume that the shifts of the oval are caused by a linear combination of three factors, $\Delta$ Psw, $\Delta B_{y}$, and $\Delta B_{z}$ (dependence on $B_{x}$ will be discussed later). Thus, we assume relations like

$$
\begin{aligned}
& D o 6=c_{P}^{o 6} \Delta P_{s w}+c_{Y}^{o 6} \Delta B_{y}+c_{Z}^{o 6} \Delta B_{z} \\
& D b 6=c_{P}^{b 6} \Delta P_{s w}+c_{Y}^{b 6} \Delta B_{y}+c_{Z}^{b 6} \Delta B_{z} \\
& D o 20=c_{P}^{o 20} \Delta P_{s w}+c_{Y}^{o 20} \Delta B_{y}+c_{Z}^{o 20} \Delta B_{z} \\
& D b 20=c_{P}^{b 20} \Delta P_{s w}+c_{Y}^{b 20} \Delta B_{y}+c_{Z}^{b 20} \Delta B_{z}
\end{aligned}
$$

The regression coefficients $\left(c_{P, Y, Z}^{o / b, 6 / 20}\right)$ are calculated using least-square minimization for each MLT sector.

Figure 2 shows the results of the multi-factor analysis for the pre-noon sector. Each row of panels relates to one of the four characteristics of the oval shift (Do6, Do20, Db6, and Db20). For instance, the top left panel shows the dependence of the residual shift,

$D_{P} o 6=D o 6-c_{Y}^{o 6} \Delta B_{y}-c_{Z}^{o 6} \Delta B_{z}$,

versus Psw. In a way, $D_{P} 06$ represents a "pure" effect of the Psw (after the effects of IMF $B_{y}$ and $B_{z}$ have been subtracted), which permits us to draw conclusions on the SW pressure effect on the mid oval shift in the first $6 \mathrm{~min}$. The other two top panels show the "pure" effects of IMF $B_{y}$ and $B_{z}$, respectively. Dots connected by vertical dashed lines indicate uncertainty on the shift values represented with asterisks. Straight lines represent the least-square linear fits of the data points. Figures 3 and 4 show the results of multi-factor analysis for the noon and post-noon sectors, respectively.

At the top of each panel, the regression coefficients are given along with the correlation coefficients between two corresponding parameters (e.g., the correlation between Psw and the residual $\mathrm{D}_{P} \mathrm{O} 6$ is given in the top left panel). When calculating the correlation, we imposed the condition that a zero interplanetary disturbance produces zero shifts of the oval. Effectively, instead of calculating the correlation between data arrays D and $\mathrm{P}$ we calculated the correlation between doubled arrays $[\mathrm{D},-\mathrm{D}]$ and $[\mathrm{P},-\mathrm{P}]$. Confidence intervals for the correlation coefficients were calculated using the following formula from Nikitin (1990, p. 270):

$\delta C_{\mathrm{corr}}=t_{\gamma} \frac{1-C_{\mathrm{corr}}^{2}}{\sqrt{n}}$,

where $n$ is the number of points (15 and 20 for D6 and D20, respectively) and $t_{\gamma}$ is the inverse standardized normal distribution (for the $95 \%$ confidence interval, $t_{\gamma}=1.96$ ).

In our analysis the correlation coefficients play the most important role. Most panels in Figs. 2-4 do not show any significant dependency of the oval motion on the interplanetary changes. There may be several reasons for that. Firstly, the simple linear model shown in Eq. (4) does not account for preconditioning of the magnetosphere. Secondly, there is a large uncertainty in the determination of the oval position due to WIC data noise, day glow removal, and weak polar cap auroras. Thirdly, more events are desirable for better statistics. Thus, we should not expect excellent dependencies and large correlations. Nevertheless, in several cases (e.g., Do20/ $B_{y}$ and Db20/ $B_{y}$ dependences at 06:00-10:00 MLT, Fig. 2) the values of the correlation coefficients are relatively large, they exceed the confidence intervals, which means that at least the direction of auroral motion can be certain (within the $95 \%$ confidence interval). In these cases, the direction of poleward (or equatorward) auroral motion along with certain changes of interplanetary parameters can be seen by eye.

The correlations shown in Figs. 2-4 are summarized in Table 2. The underlined bold numbers are the coefficients which values exceed the confidence intervals. Positive (negative) correlation values correspond to poleward (equatorward) motion of the auroras. The following conclusions can be made from data in Table 2:

1. A Psw increase causes an equatorward shift of the mid oval position at $\mathrm{t}_{0}+6 \mathrm{~min}$ in the 14:00-18:00 MLT sector.

2. A Psw increase causes a poleward shift of PAB at $\mathrm{t}_{0}+6$ min in the 14:00-18:00 MLT sector and later, at $\mathrm{t}_{0}+20 \mathrm{~min}$, in the 06:00-10:00 MLT sector.

3. An IMF $B_{y}$ increase causes an equatorward shift of the mid oval position and PAB at $\mathrm{t}_{0}+20 \mathrm{~min}$, in 06:00 14:00 MLT sectors.

4. IMF $B_{z}$ within $20 \mathrm{~min}$ does not affect the position of dayside auroral oval (in the 06:00-18:00 MLT sector).

Finally, we performed a similar analysis including IMF $B_{x}$ changes in Eq. (4). This analysis has not revealed any effect of IMF $B_{x}$. However, the IMF $B_{x}$ changes were essentially smaller than ones in the other IMF components (less than $3 \mathrm{nT})$. Hence, from our data it is impossible to conclude on the role of changes in IMF $B_{x}$. 

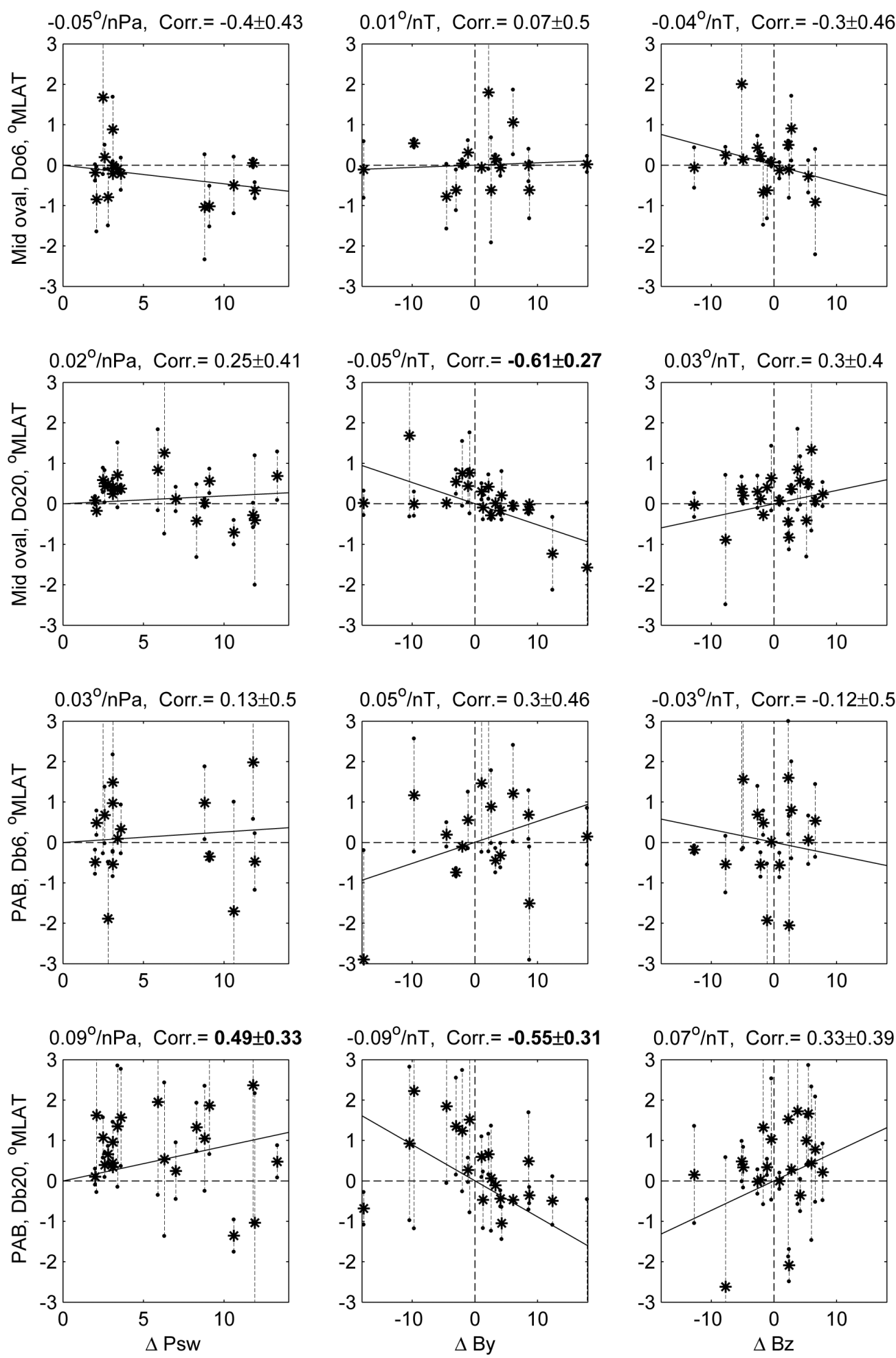

$M L T=6-10$

Fig. 2. Shift of the oval and PAB in 06:00-10:00 MLT sector versus Psw, IMF $B_{y}$, and IMF $B_{z}$. Dots connected by vertical dashed lines show uncertainty intervals for the data points. 

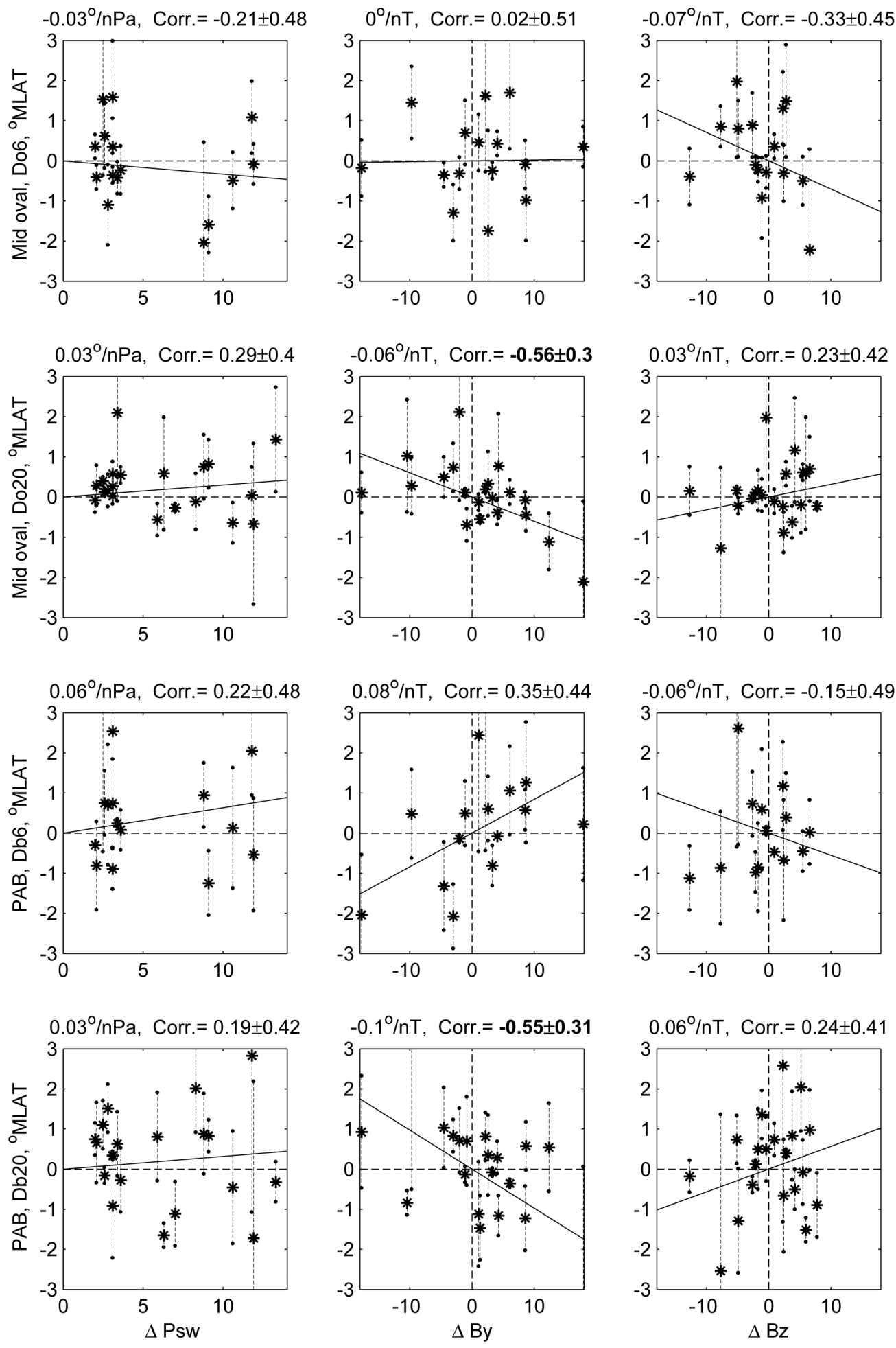

MLT $=10-14$

Fig. 3. Same as Fig. 2, but for the 10:00-14:00 MLT sector. 

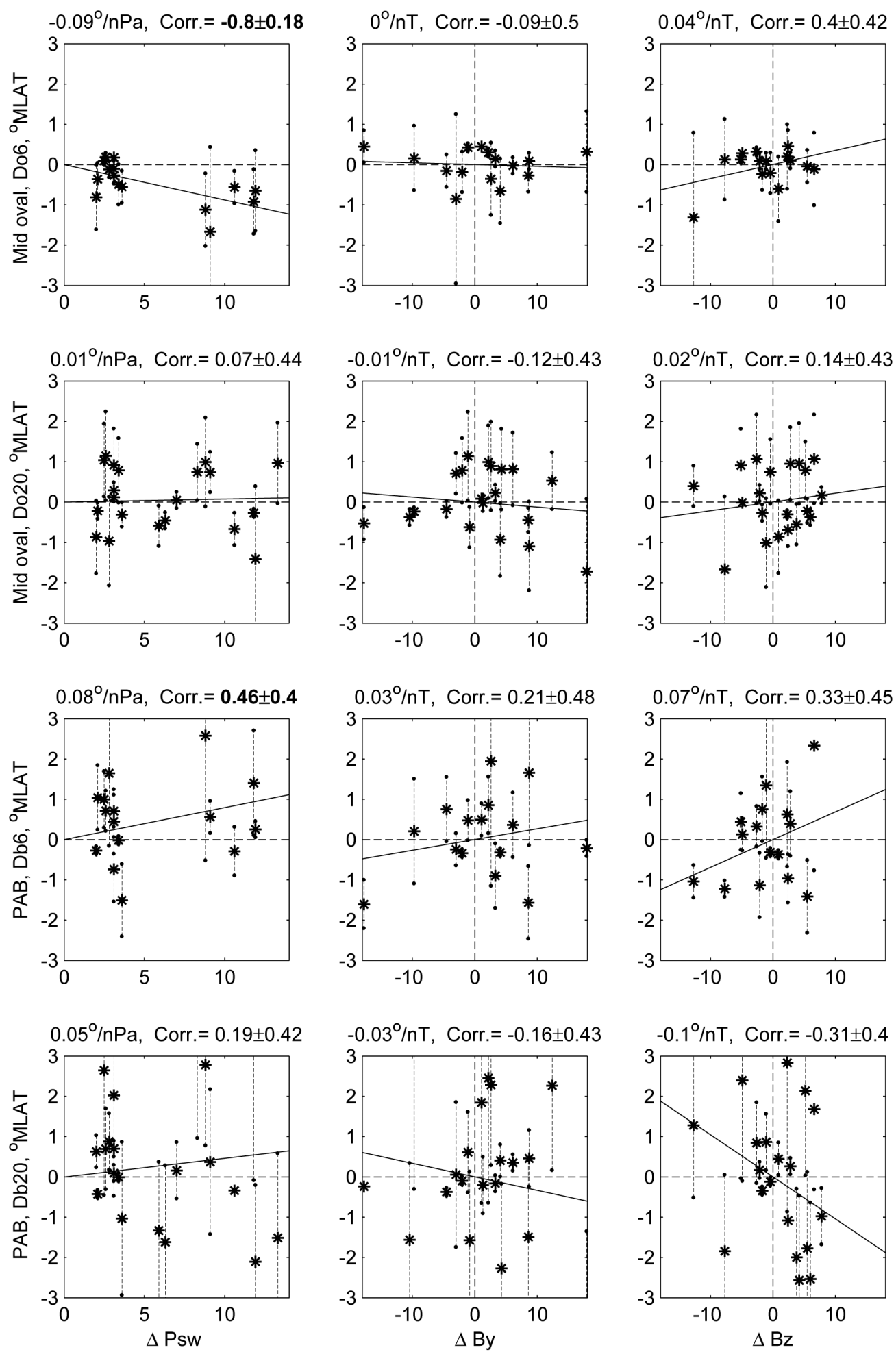

$M L T=14-18$

Fig. 4. Same as Fig. 2, but for the 14:00-18:00 MLT sector. 
Table 2. Correlation coefficients.

\begin{tabular}{|c|c|c|c|c|c|c|c|c|c|}
\hline $\begin{array}{l}\text { Parameter } \\
\text { MLT sector }\end{array}$ & 14:00-18:00 & $\begin{array}{c}\text { Psw } \\
10: 00-14: 00\end{array}$ & 06:00-10:00 & 14:00-18:00 & $\begin{array}{c}\text { IMF } B_{y} \\
10: 00-14: 00\end{array}$ & 06:00-10:00 & 14:00-18:00 & $\begin{array}{c}\text { IMF } B_{z} \\
10: 00-14: 00\end{array}$ & 06:00-10:00 \\
\hline Mid oval 6 min & -0.80 & -0.21 & -0.40 & -0.09 & 0.02 & 0.07 & -0.03 & 0.33 & -0.30 \\
\hline Mid oval $20 \mathrm{~min}$ & $\overline{0.07}$ & 0.29 & 0.25 & -0.12 & -0.56 & -0.61 & 0.14 & 0.23 & 0.30 \\
\hline PAB 6 min & $\underline{0.46}$ & 0.22 & 0.13 & 0.21 & 0.35 & 0.30 & 0.34 & -0.15 & -0.12 \\
\hline PAB $20 \mathrm{~min}$ & 0.19 & 0.19 & $\underline{0.49}$ & -0.16 & -0.55 & -0.55 & -0.29 & 0.24 & 0.33 \\
\hline
\end{tabular}

\section{Interpretation and discussion}

\subsection{Effect of Psw: midday sub-auroral patches}

The most important effect of solar wind pressure increase is the equatorward shift of the mid oval at $t_{0}+6$ min in the 14:00-18:00 MLT sector (see Fig. 4, top left panel). In the other MLT sectors, motion of the mid oval tends to be equatorward, as well, which corresponds to negative correlation coefficients. This equatorward shift is consistent with the result shown by Liou et al. (2002) who observed sudden brightening of the auroral patch in the midday (10:00-14:00 MLT) sub-auroral zone (above $65^{\circ}$ MLAT) caused by the SI. The patches were named Midday Sub-auroral Patches (MSPs) and identified by the following characteristics: lifetime of MSP is short ( 5-6 min), after brightening MSP widened and/or moved equatorward, and they are separated from the main (pre-existing) auroral oval. The magnetospheric source region for MSPs was identified in the central plasma sheet (CPS). It has been suggested that sudden compression of the Earth's magnetic field may change the pitch angles of a preexisting population of CPS particles (e.g., through cyclotron instability), which consequently fall into the loss cone and produce auroral emission. The MSP is well seen in the WIC frames from $t_{0}+1$ to $t_{0}+6$ (Fig. 1) in the noon-postnoon sector equatorward of the middle auroral oval. At $t_{0}+8$, the MSP has faded.

The MSP observed in Liou et al. (2002) were caused mainly by electron precipitation from CPS. On the other hand, Hubert et al. (2003) and Fuselier et al. (2004) studied shock-induced proton auroras and found phenomena similar to MSP called "subauroral proton flashes" (Hubert et al., 2003) or "transient dayside subauroral proton precipitation" (Fuselier et al., 2004). These shock-triggered proton auroras have the same temporal characteristics (response and life times) as electron MSPs, occur in the near-noon MLT sectors and are separated from the main auroral oval. However, they reside at lower latitudes (down to $60^{\circ}$ MLAT), which corresponds to the region of the plasmapause (Fuselier et al., 2004). The mechanism suggested for proton precipitation is pitch-angle scattering due to ion-cyclotron instability triggered by a SI. Therefore, the physics of sub-auroral electron and ion precipitation triggered by a SI (electron- and ioncyclotron instabilities, respectively) are similar in many re- spects. In the present study we do not separate electron and ion contributions to the shock-induced aurora observed by WIC. For our analysis, it is essential that sub-auroral patches (referred to MSP) are generated by a SWP jump and result in an equatorward shift of the mid oval as defined by Eq. (2). Hence, the correlation obtained in the present study agrees with the earlier studies.

Our observations suggest that the most significant MSP effect is in the 14:00-18:00 MLT sector. Fuselier et al. (2004) determined that the local time occurrence of proton precipitation is correlated with the orientation of the pressure pulse front in the solar wind. In the present study, on average, the pressure front hits the magnetosphere near noon. Thus, other factors should be taken into account. Indeed, Fuselier et al. (2004) pointed out reasons for which proton precipitation occurs preferably in the dusk side. Firstly, cold plasma is typically farther away from the Earth on the dusk side (plasmasphere bulge). Secondly, the hot ring current proton anisotropy peaks in the dusk side near the magnetopause. Thus, more favorable conditions occur on the dusk side for the ion-cyclotron instability with subsequent proton precipitation.

\subsection{Effect of Psw: high-latitude auroras}

The second clear effect of the solar wind pressure increase is the poleward shift of the PAB. As we discussed above, such a shift indicates auroral intensification or generation of new auroras at high latitudes (in the vicinity of the PC boundary). For the first $6 \mathrm{~min}$ high-latitude auroral intensification is most prominent in the post-noon 14:00-18:00 MLT sector and later, from 6 to $20 \mathrm{~min}$ after the SI, intensification occurs in the pre-noon 06:00-10:00 MLT sector (see Table 2).

This effect is similar to one documented by Kozlovsky et al. (2005). They used data from the IMAGE satellite along with ground optical observations on 7 December 2000 (event 8 in Table 1). In this case, the SI caused new discrete auroral forms which appeared in the poleward part of the auroral oval previously filled with diffuse auroras. Consequently, the region of discrete auroras expanded poleward. Possible generation mechanisms for the discrete auroras launched by the SI can be the interchange instability and the eigenmode toroidal Alfvén oscillations (see (Kozlovsky et al., 2005) for more details). Poleward expansion occurred 

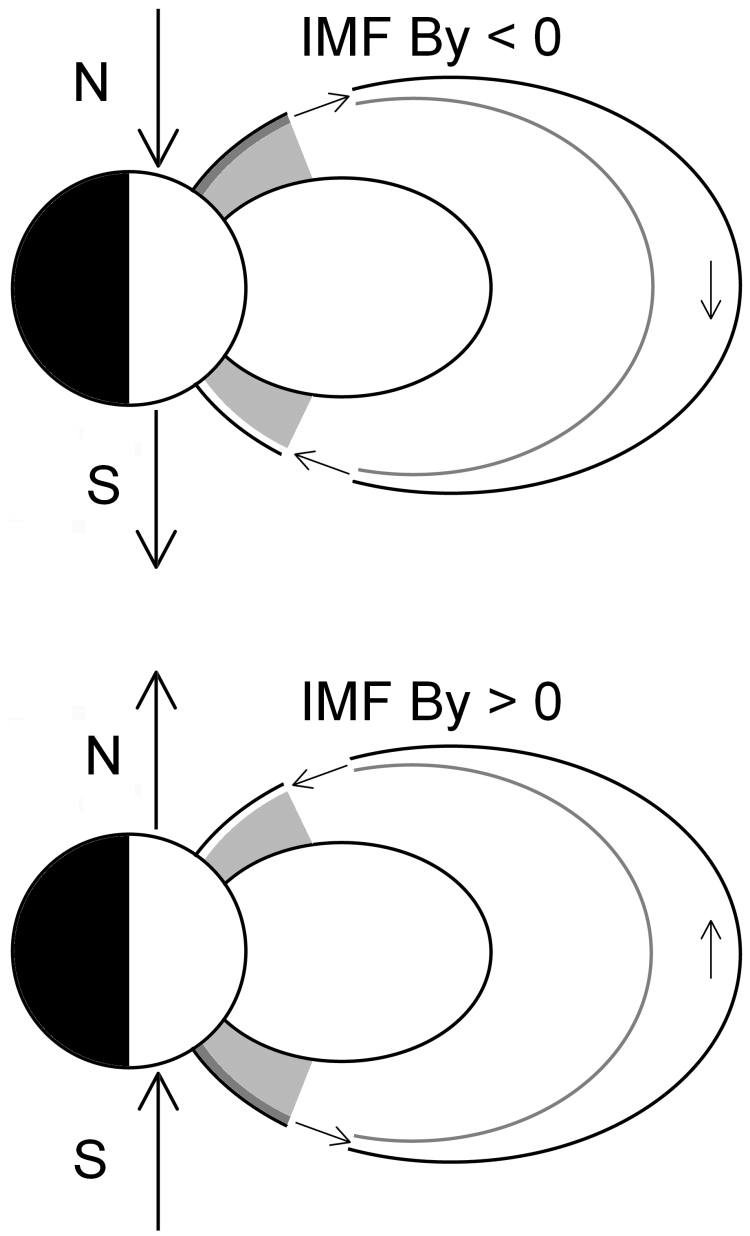

Fig. 5. The field-aligned current system generated by IMF $B_{y}$ (the currents are indicated by arrows): The field-aligned currents flow from the solar wind to the open polar cap ionosphere then at the boundary of open/closed field lines (within a thin layer of the closed magnetosphere) they transfer into the opposite hemisphere, and exit the ionosphere from the opposite polar cap. In the inter-hemispheric FAC, a field-aligned potential drop should arise, which can affect on the auroral intensity (indicated by shaded areas). In the upward FAC, the field-aligned potential drop increases the energy of the precipitating auroral electrons, which implies intensification of the most poleward auroras and can be seen as a poleward expansion of the auroral oval (top panel). In opposite, the downward FAC associated with IMF $B_{y}>0$ suppress the most poleward precipitation that means a shift of PAB to lower latitudes (bottom panel).

first in the post-noon MLT sector, where in this case the pressure pulse hit the magnetosphere and then it was seen in the pre-noon MLT sectors, which suggested that the disturbance "propagated" to the west (with a velocity of $\sim 7 \mathrm{~km} / \mathrm{s}$ at the ionospheric altitude) from the 15:00 MLT sector where the SI encountered the magnetosphere. The present statistical study also suggests that high-latitude auroras first $(\mathrm{t}<6 \mathrm{~min})$ appeared in the post-noon sector, and later $(\mathrm{t}=6-20 \mathrm{~min})$ occurred in the morning sector. However, pressure front orien- tation for the studied cases does not show preferences in the post-noon sector. Thus, we can not attribute this effect to the pressure front orientation. The explanation of this result may be in the scope of future studies.

One more possible reason for the poleward shift of PAB may be a poleward motion of open-closed field boundary (OCB), which was shown by Boudouridis et al. (2004) on the base of particle precipitation data from DMSP satellites. They explained this by the increase of magnetotail reconnection during magnetosphere compression, which is the most significant on the nightside during southward IMF. Simultaneous enhancement of the magnetospheric convection may lead to a relative fast transport of the newly-closed magnetic flux tubes to the dayside and corresponding OCB motion poleward. Thus, both the generation of high-latitude auroras and the OCB motion may contribute to the PAB motion, but the data used in the present study do not allow distinguishing between these two effects.

The generation of high-latitude auroras should also cause a poleward motion of the mid auroral oval. Midday sub-auroral patches result in an equatorward shift of the mid oval during the first 6 min after the SI, but they fade afterwards, which may be seen as poleward mid oval motion. Indeed, 6-20 min after the SI the Psw-related motion of the mid oval tends to be poleward (positive correlation coefficients). That may be due to both high-latitude auroral intensification and MSP fading.

\subsection{Effects of IMF $B_{y}$}

Among our results, the most distinct is the IMF $B_{y}$ effect on the positions of dayside PAB and mid oval 6-20 min after a SI. It can be interpreted as intensification of the most poleward auroras due to IMF $B_{y}$ turning negative (when the oval and PAB are moving toward the pole), whereas IMF $B_{y}$ turning positive leads to fading of the most poleward auroras (observed as a shift of the PAB towards the equator).

This effect can be explained in frame of the earlier study (Kozlovsky et al., 2003b) where the high-latitude fieldaligned current (FAC) system associated with IMF $B_{y}$ was considered. Due to the solar wind motion with respect to the Earth, the IMF $B_{y}$ component is associated with a northsouth electric field in the undisturbed solar wind (on open field lines). Through the well-conducting ionosphere, the footprints of the open field lines are electrically connected to the footprints of the closed magnetic field lines, so that the electric connection occurs between the open field lines over the southern polar cap and the open field lines over the northern polar cap. Thus, IMF $B_{y}$ generates a voltage between the two polar caps, which drives field-aligned currents between the hemispheres (Fig. 5). The field-aligned currents flow from the solar wind to the open polar cap ionosphere, then at the boundary of open/closed field lines (in a thin layer of the closed magnetosphere) they transfer to the opposite hemisphere, and finally the FACs exit the ionosphere from the opposite polar cap. The relative position of the up- and 
downward FACs depends on the IMF $B_{y}$ sign: for a negative IMF $B_{y}$, upward FAC in the Northern Hemisphere is located equatorward of downward FAC (top panel in Fig. 5), for positive IMF $B_{y}$, the positions of the currents are reversed (bottom panel in Fig. 5).

The inter-hemispheric FACs occur inside a layer just equatorward of the polar cap boundary. The latitudinal width of this region depends on IMF $B_{y}$ and ionospheric conductance, and can be of the order of 1-2 degrees in latitude. In this region a field-aligned potential difference should arise to prevent the magnetic flux tube twisting associated with the interhemispheric FAC (Kozlovsky et al. 2003b). In the upward FAC (for IMF $B_{y}<0$ in the Northern Hemisphere), the fieldaligned potential drop increases the energy of precipitating auroral electrons, which implies intensification of the most poleward auroras (indicated by shaded areas in Fig. 5) and can be seen as a poleward expansion of the auroral oval (top panel in Fig. 5). In opposite, the downward FAC associated with IMF $B_{y}>0$ suppress the most poleward precipitations, which means a shift of PAB to lower latitudes (bottom panel in Fig. 5). Thus, IMF $B_{y}$ can affect the PAB position. In the Northern Hemisphere, an IMF $B_{y}$ increase from negative to positive value leads to a shift of PAB to lower latitudes. In the Southern Hemisphere the effect is reversed.

We have obtained that IMF $B_{y}$ changes take 6-20 min to affect high-latitudes auroral distributions, which is a characteristic time for the field-aligned current system to setup. Obviously, the IMF $B_{y}$ effect does not relate to SWP changes, i.e., it takes place also in the cases when IMF $B_{y}$ changes occur during constant SWP. However, if a solar wind pressure change is associated with change in IMF $B_{y}$, the effects of IMF $B_{y}$ should be taken into account.

Finally, one should mention that no significant motion of the dayside auroral oval was observed in association with IMF $B_{z}$ changes. However, we studied only first 20 min after the changes, whereas the IMF $B_{z}$-related effects may be on a larger time scale. Indeed, Kozlovsky and Kangas (2001) studied dynamics of the pre-noon auroral oval and found that IMF $B_{z}$ turning southward resulted in the equatorward oval motion starting $27 \pm 7 \mathrm{~min}$ after the IMF $B_{z}$ turning at the subsolar point. Thus, the auroral oval response time to IMF $B_{z}$ changes is most likely greater than $20 \mathrm{~min}$.

\section{Summary and conclusion}

WIC global auroral images of the IMAGE satellite were used for a statistical (on the base of 20 cases) study of the position changes of the dayside auroras 20 min after abrupt increases in the solar wind ram pressure. Contributions from the IMF changes associated with the shock have also been investigated. The effects of the IMF and pressure changes were separated using multi-factor correlation analysis.

1. The most important effect of solar wind pressure increase is the auroral intensification equatorward of the mid auroral oval during 6 min after a SI, which is the most prominent in post-noon MLT sector. This intensification is consistent with midday sub-auroral patches (MSPs) equatorward of the main auroral oval. The MSPs were introduced by Liou et al. (2002) and Hubert et al. (2003). They suggested that a sudden compression of the Earth's magnetic field may change the pitch angles of a pre-existing population of magnetospheric electrons and protons, which consequently fall into the loss cone and produce the MSP auroral emission.

2. The second effect of solar wind pressure increase is the auroral intensification at high latitudes in the vicinity of the PC boundary. For the first 6 min after a SI the highlatitude auroral intensification is most prominent in the post-noon 12:00-18:00 MLT sector and at 6 to $20 \mathrm{~min}$ after the SI intensification occurs in the pre-noon 06:0012:00 MLT sector.

3. The most obvious effect of the IMF changes is the IMF $B_{y}$ effect on the dayside auroral distribution from 6 to 20 min after the SI, which is interpreted as the intensification of the most poleward auroras due to IMF $B_{y}$ turning negative, whereas IMF turning positive leads to fading of the most poleward auroras. Such an effect is consistent with the IMF $B_{y}$-related system of fieldaligned currents.

4. No significant motion of the dayside auroral oval was observed in association with IMF $B_{z}$ changes. Most likely, the auroral oval response time to IMF $B_{z}$ changes is larger than $20 \mathrm{~min}$.

Acknowledgements. The IMAGE-FUV investigation was supported by NASA through SWRI subcontract number 83820 at the University of California, Berkeley, contract NAS5-96020. We thank the Coordinated Data Analysis Web (CDAWeb) and the data providers D. J. McComas (Southwest Research Institute) and N. Ness (Bartol Research Institute) for the ACE Solar Wind Experiment and magnetic field data, respectively, R. Lepping (NASA/GSFC) for the Wind magnetic field data and K. Ogilvie (NASA GSFC) for the Wind solar wind data. Data of the magnetic observatories Guimar, Kanoya, Honolulu, and Tondano were obtained through the SPIDR data system. We thank N. Østgaard at the University of Bergen for useful discussion and L. Sangalli at the University of Calgary for help in paper writing. The study was supported by the Academy of Finland.

Topical Editor I. A. Daglis thanks L. Lyons and A. Boudouridis for their help in evaluating this paper.

\section{References}

Boudouridis, A., Zesta, E., Lyons, R., Anderson, P. C., and Lummerzheim, D.: Effect of solar wind pressure pulses on the size and strength of the auroral oval, J. Geophys. Res., 108(A4), 8012, doi:10.1029/2002JA009373, 2003. 
Boudouridis, A., Zesta, E., Lyons, R., Anderson, P. C., and Lummerzheim, D.: Magnetospheric reconnection driven by solar wind pressure fronts, Ann. Geophys., 22, 1367-1378, 2004, http://www.ann-geophys.net/22/1367/2004/.

Brittnacher, M., Wilber, M., Fillingim, M., Chua, D., Parks, G., Spann, J., and Germany, G.: Global auroral response to a solar wind pressure pulse, Adv. Space Res., 25, 1377-1385, 2000.

Fuselier, S. A., Gary, S. P., Thomsen, M. F., Claflin, E. S., Hubert, B., Sandel, B. R., and Immel, T.: Generation of transient dayside subauroral proton precipitation, J. Geophys. Res., 109, A12227, doi:10.1029/2004JA010393, 2004.

Hubert, B., Gérard, J. C., Fuselier, S. A., and Mende, S. B.: Observation of dayside subauroral proton flashes with the IMAGE-FUV imagers, Geophys. Res. Lett., 30(3), 1145 , doi:10.1029/2002GL016464, 2003.

Khan, H. and Cowley, S. W. H.: Observations of the response time of high-latitude ionospheric convection to variations in the interplanetary magnetic field using EISCAT and IMP-8 data, Ann. Geophys., 17, 1306-1335, 1999. http://www.ann-geophys.net/17/1306/1999/.

Kozlovsky, A. and Kangas, J.: Characteristics of the post-noon auroras inferred from EISCAT radar measurements, J. Geophys. Res., 106, 1817-1834, 2001.

Kozlovsky, A., Safargaleev, V., Jussila, J., and Koustov, A.: Prenoon high-latitude auroral arcs as a manifestation of the interchange instability, Ann. Geophys., 21, 2303-2314, 2003a.

Kozlovsky, A., Turunen, T., Koustov, A., and Parks, G.: IMF By effects in the magnetospheric convection on closed magnetic field lines, Geophys. Res. Letters, 30(24), 2261, doi:10.1029/2003GL018457, 2003b.

Kozlovsky, A., Safargaleev, V., Østgaard, N., Turunen, T., Koustov, A., Jussila, J., and Roldugin, A.: On the motion of dayside auroras caused by a solar wind pressure pulse, Ann. Geophys., 23, 509-521, 2005, http://www.ann-geophys.net/23/509/2005/.

Liou, K., Wu, C.-C., Lepping, R. P., Newell, P. T., and Meng, C.-I.: Midday sub-auroral patches (MSPs) associated with interplanetary shocks, Geophys. Res. Lett., 29(16), 1771, doi:10.1029/2001GL014182, 2002.

Lyons, L. R., Lee, D.-Y., Wang, C.-P., and Mende, S. B.: Global auroral responses to abrupt solar wind changes: Dynamic pressure, substorm, and null events, J. Geophys. Res., 110, A08208, doi:10.1029/2005JA011089, 2005.
Mende, S. B., Heetderks, H., Frey, H. U., Lampton, M., Geller, S. P., Habraken, S., Renotte, E., Jamar, C., Rochus, P., Spann, J., Fuselier, S. A., Gerard, J.-C., Gladstone, R., Murphree, S., and Cogger, L.: Far ultraviolet imaging from the IMAGE spacecraft. 1. System design, Space Sci. Rev., 91(1-2), 243-270, 2000a.

Mende, S. B., Heetderks, H., Frey, H. U., Lampton, M., Geller, S. P., Abiad, R., Siegmund, O. H. W., Tremsin, A. S., Spann, J., Dougani, H., Fuselier, S. A., Magoncelli, A. L., Bumala, M. B., Murphree, S., and Trondsen, T.: Far ultraviolet imaging from the IMAGE spacecraft. 2. Wideband FUV imaging, Space Sci. Rev., 91(1-2), 271-285, 2000b.

Meurant, M., Gérard, J.-C., Hubert, B., Blockx, C., Østgaard, N., and Mende, S. B.: Dynamics of global scale electron and proton precipitation induced by a solar wind pressure pulse, Geophys Res. Lett., 30(20), 2032, doi:10.1029/2003GL018017, 2003.

Meurant, M., Gérard, J.-C., Blockx, C., Hubert, B., and Coumans, V.: Propagation of electron and proton shock-induced aurora and the role of the interplanetary magnetic field and solar wind, J. Geophys. Res., 109, A10210, doi:10.1029/2004JA010453, 2004.

Meurant, M., Gérard, J.-C., Blockx, C., Coumans, V., Hubert, B., Connors, M., Lyons, L. R., and Donovan, E.: Comparison of intense nightside shock-induced precipitation and substorm activity, J. Geophys. Res., 110, A07228, doi:10.1029/2004JA010916, 2005.

Nikitin, A. A.: Probability and statistical methods of treatment and interpretation of the results of geophysical observations, in: Computing mathematics and techniques in the exploration geophysics, edited by: Dmitriev, V. I., pp. 235-339, Nedra, Moscow (In Russian), 1990.

Trondsen, T. S., Lyatsky, W., Cogger, L. L., and Murphree, J. S.: Interplanetary magnetic field By control of dayside auroras, J. Atmos. Terr. Phys., 61, 829-840, 1999.

Zhou, X. and Tsurutani, B. T.: Rapid intensification and propagation of the dayside aurora: Large scale interplanetary pressure pulses (fast shocks), Geophys. Res. Lett., 26, 1097-1100, 1999.

Zhou, X. and Tsurutani, B. T.: Interplanetary shock triggering of nightside geomagnetic activity: Substorms, pseudobreakups, and quiescent events, J. Geophys. Res., 106(A9), 18 957-18968, 2001. 